\title{
Clinical Presentation and Genetic Variants in Autoinflammatory Diseases: Results From the German anti-IL1 Registry for Orphan Diseases (GARROD)
}

Norbert Blank ( $\nabla$ norbert.blank@med.uni-heidelberg.de)

University Hospital Heidelberg: UniversitatsKlinikum Heidelberg https://orcid.org/0000-0002-94191690

Ina Kötter

University Hospital Hamburg-Eppendorf: Universitatsklinikum Hamburg-Eppendorf

Hans-Peter Tony

University Hospital Wurzburg: Universitatsklinikum Wurzburg

Jürgen Rech

Erlangen University Hospital: Universitatsklinikum Erlangen

Karoline Krause

Charite University Hospital Berlin: Charite Universitatsmedizin Berlin

Birgit Köhler

Karlsruhe Hospital Department of Internal Medicine: Stadtisches Klinikum Karlsruhe gGmbH

Medizinische Klinik I

\section{Dorothee Kaudewitz}

University Hospital Heidelberg: UniversitatsKlinikum Heidelberg

\section{Martin Nitschke}

Universitätsklinikum Schleswig-Holstein - Campus Lübeck: Universitatsklinikum Schleswig Holstein -

Campus Lubeck

Christian Haas

Universitatsklinikum Giessen und Marburg - Standort Marburg

Hanns-Martin Lorenz

University Hospital Heidelberg: UniversitatsKlinikum Heidelberg

Martin Krusche

Charite University Hospital Berlin: Charite Universitatsmedizin Berlin

\section{Research Article}

Keywords: Anakinra, Familial Mediterranean Fever, Cryopyrin-associated periodic syndrome, Tumor Necrosis Factor Receptor-Associated Periodic Syndrome, Adult-onset Still's disease 
Posted Date: March 15th, 2021

DOI: https://doi.org/10.21203/rs.3.rs-293402/v1

License: (c) (i) This work is licensed under a Creative Commons Attribution 4.0 International License. Read Full License 


\section{Abstract}

Background: To investigate the clinical presentation and genetic variants in patients of the German antiIL1 registry for autoinflammatory orphan diseases (GARROD) between 2013-2019.

Methods: Multicenter, retrospective analysis of demographic, clinical and genetic data of 231 patients with autoinflammatory diseases (AID) who received anti-IL1 targeted therapy.

Results: Inflammatory attacks started before the age of 18 years in $44 \%$ of the patients. Symptom onset was on average 17.8 years prior to the initiation of anti-IL 1 targeted therapy. AID spectrum comprised familial Mediterranean fever (FMF; $n=71 ; 30.7 \%$ ), cryopyrin-associated periodic syndromes (CAPS; $n=43$; $18.6 \%)$, adult-onset Still's disease (AOSD, $n=32 ; 13.8 \%)$ or other AID ( $n=85 ; 36.8 \%)$. Monogenetic AID were diagnosed by clinical symptoms. Genetic analyses confirmed the diagnosis in $65 \%$ of patients with FMF, $14 \%$ with CAPS and $47 \%$ with TNF receptor-associated periodic syndrome (TRAPS). Heterozygous MEFV variants and variants of unknown significance (VUS) were detected in $23 \%$ of patients with FMF, $51 \%$ with CAPS and $47 \%$ with TRAPS. Patients with VUS were older at disease onset, indicating a milder phenotype.

Twenty-nine patients (12.5\%) had secondary AA amyloidosis at initiation of anti-IL1 therapy. Primary Diseases were FMF $(n=22)$, TRAPS $(n=1)$ and idiopathic AA amyloidosis $(n=6)$.

Conclusions: Molecular genetic analyses might substantiate the clinical diagnosis of a monogenetic AID. In the absence of a known pathogenic variant, the functional role of VUS can be discussed. Our data support the concept of a variable penetrance of VUS, leading to late-onset AID.

Anti-IL1 targeted drugs are used to control systemic inflammation, prevent disease flares and AA amyloidosis.

\section{Key Messages}

- AID comprise monogenetic and polygenetic diseases

- a significant proportion of patients with presumably monogenetic AID do not receive genetic confirmation

- genetic variants correlate with disease onset in patients with monogenetic AID

- up to $56 \%$ of adult onset AID patients require anti-IL1 treatment

- the long period between onset of AID attacks and IL-1 targeted treatment highlights the urgent need for improvement for the management of AID

\section{Introduction}


The increasing knowledge about autoinflammatory diseases (AID) has changed our view of systemic inflammation over the recent decades. FMF is the most prevalent AID world-wide and colchicine is the standard therapy to prevent FMF attacks and AA amyloidosis. Classification criteria were developed for familial mediterranean fever (FMF) [1], Cryopyrin associated periodic syndrome (CAPS) [2] and TNF Receptor associated periodic syndrome (TRAPS) [3]. Although genetic variants for FMF, CAPS and TRAPS are known, these variants are neither essential for AID diagnosis nor have they been included in the classification criteria.

Patients with systemic onset of juvenile idiopathic arthritis (soJIA, classic Still's disease) and adult onset Still's disease (AOSD) are considered to be polygenetic diseases representing two manifestations within a disease spectrum [4]. The demonstration of genetic variants in a subgroup of patients with AOSD suggests a genetic overlap between monogenetic and polygenetic disease with similar clinical features [5]. The clinical and laboratory features of AOSD were classified previously [6, 7]. Recently, AOSD was characterized as a disease comprising a broad spectrum of manifestations and potentially lifethreatening complications [8].

Investigations on the pathophysiological role of the inflammasome led to the recognition of interleukin1 beta (IL-1 $\beta$ ) as the key cytokine in monogenetic and polygenetic AID. Blocking of IL-1 $\beta$ is an effective treatment option for monogenetic and polygenetic AID $[9,10]$. The IL1-receptor antagonist anakinra (AKN) inhibits the binding of IL-1 $\beta$ to the IL1-receptor. The monoclonal antibody canakinumab (CAN) inhibits IL$1 \beta$ activity by binding to IL-1 $\beta$. Recently, AKN [11] and CAN [12] showed efficacy for AOSD treatment with and without macrophage activation syndrome.

The German Anti-IL1 treatment RegistRy in Orphan Diseases (GARROD) was initiated in 2013 when it became evident that blocking IL-1 $\beta$ is effective in AID $[6,7]$. The GARROD registry is a multicentric retrospective analysis of adult patients with autoinflammatory diseases (AID) which were treated with AKN or CAN on an individual basis. The aim of the registry was to provide a research platform to gather and analyze data on the health care of patients with AID as well as to collect information on the safety and efficacy of anti-IL1 $\beta$ treatment.

We compared the clinical diagnoses with the results of genetic analyses in patients with monogenetic and polygenetic AID.

\section{Material \& Methods}

\section{Patient data:}

The GARROD registry was initiated in October 2013 at the University of Heidelberg. It was established as a multi-center registry and until December 2018 various institutions in Germany contributed depersonalized data to GARROD. The registry included a consecutive cohort of patients with AID who were treated with AKN or CAN for longer than two months. The purpose of GARROD was to gather and analyze data about diagnoses, safety and efficacy of anti-IL1 $\beta$ treatment in patients with AID. The project 
was approved by the ethical review committee at the University of Heidelberg (S-103/2013) and by the local ethical committees at the participating centers.

\section{Clinical diagnoses of AID and genetic analysis}

FMF [1], CAPS [2], TRAPS [3] and AOSD [4, 5] were diagnosed by clinical AID experts, according to the previously published classification criteria. Patients with non-classified AID, treatment-refractory gout, pleuro-pericarditis, Schnitzler's syndrome, idiopathic AA amyloidosis and other rare diseases according to expert opinion were also included in this registry. Patients with the clinical diagnosis of a monogenetic AID were screened for variants in MEFV(FMF), NLRP3 (CAPS) and TNFRSF1A (TRAPS). Genetic analyses were conducted at several commercial laboratories.

Single nucleotide polymorphisms (SNP) with a minor allele frequency (MAF) of $\geq 1 \%$ in the general population were considered as a variant of unknown significance (VUS) or as benign variant. The Infevers database [13, last access 04 Jan 2021] served as a reference to evaluate the functional role of single variants. The nomenclature of genetic variants followed the current guidelines of the Human Genome Organisation (HUGO), Human Genome Nomenclature Committee (HGNC), Human Genome Variation Society (HGVS) and the Infevers database [13]. The MAF was derived from the National Center for Biotechnology Information (NCBI) SNP data base (https://www.ncbi.nlm.nih.gov/pubmed).

The MEFV variant p.Arg202GIn (R202Q, rs224222, MAF 0.136) was considered as benign polymorphism. The MEFV variants p.Glu148GIn (E148Q, rs3743930, MAF 0.126), p.GIn230Lys (E230K, rs104895080, MAF 0.00001), p.Pro369Ser (P369S, rs11466023, MAF 0.02), p.Arg408GIn (R408Q, rs11466023, MAF 0.02), p.lle591Thr (I591T, rs11466045, MAF 0.004) and p.Ala744Ser (A744S, rs61732874, MAF 0.002) were considered as VUS. The MEFV variants p.Phe479Leu (F479L, rs104895083, MAF 0.00001), p.Met680lle (M680I, rs28940580, MAF 0.00006), p.Met694Val (M694V, rs61752717, MAF 0.0002), p.Lys695Arg (K695R, rs104895094, MAF 0.002), p.Val726Ala (V726A, rs28940579, MAF 0.0002) and p.Arg761His $(\mathrm{R} 761 \mathrm{H}, \mathrm{rs} 104895097, \mathrm{MAF}$ 0.0002) were considered as pathogenic MEFV variants.

NLRP3 variants p.Arg137His (R135H, rs138946894 MAF 0.0002), p.Val200Met (V198M, rs121908147, MAF 0.004), p.Leu362GIn (L360Q, unknown), p.Arg490Lys (R488K, rs145268073, MAF 0.0002), p.Arg556Leu (R554L, unknown), p.Phe581Tyr (F579Y, unknown), p.Pro651Ser (P649S, unknown) and p.GIn705Lys (Q703K, rs35829419, MAF 0.02) were considered as VUS. Pathogenic NLRP3 variants were p.Arg262Trp (R260W, rs121908150), p.Asp305Asn (D303N, 121908153), p.Thr350Met (T348M, rs151344629), p.Ala441Val (A439V, rs121908146) and p.Gly571Arg (G569R, rs121908151).

The TNFRSF1A variant p.Arg121GIn (R92Q, rs4149584; MAF 0.006) was considered as VUS. Pathogenic TNFRSF1A variants were p.Tyr49Cys (Y20C; unknown), p.Tyr49His (Y20H; unknown), p.Cyc84Arg (C55R; unknown), p.Thr79Met (T50M; rs104895219) and p.lle199Asn (I170N; unknown).

\section{Anti-IL1 targeted medication:}


AKN was initiated using $100 \mathrm{mg}$ sc daily. Patients with renal failure started with AKN 100mg sc 3 times a week. Patients with severe local reactions to AKN reduced their schedule to AKN every 2 to 3 days until local reactions disappeared. Patients with partial control of the attacks used AKN 100mg bid during an attack and $100 \mathrm{mg}$ every day for maintenance.

CAN was initiated using $150 \mathrm{mg}$ sc. every 8 weeks. Patients with a transient response for 4 to 8 weeks reduced the intervals to $150 \mathrm{mg}$ every 4 weeks. Patients with a partial response to $150 \mathrm{mg}$ used CAN $300 \mathrm{mg}$ every 4 to 8 weeks to maximize the response and to achieve remission.

Some patients who had an inadequate response or intolerance to AKN were switched to CAN.

\section{Concomitant medication:}

Concomitant medication was continued if the patient reported at least partial benefits and additional effectivity compared IL-1 $\beta$ inhibitory monotherapy. FMF patients continued with colchicine if they tolerated at least $0.5 \mathrm{mg}$ per day or more. The majority of FMF patients took $2 \mathrm{mg}$ colchicine per day. Patients with other AID had concomitant colchicine if they reported any benefit before the addition of AKN. Patients with AOSD, TRAPS and pleuro-pericarditis used pulses of prednisolone $40 \mathrm{mg}$ and tapering to control inflammatory attacks.

\section{Diagnosis of amyloidosis:}

All patients were screened for renal insufficiency, proteinuria and myocardial hypertrophy as potential indicators for amyloidosis [14]. If functional organ abnormalities were detected, the affected organs were biopsied. The diagnosis of amyloidosis was based on a positive Congo red staining and a yellow-green birefringence on polarization microscopy. The type of AA amyloid was confirmed by using immunohistochemistry [15].

\section{Statistics:}

Statistical analyses were performed using the Statistical Package for the Social Sciences (IBM SPSS Version 19) and the R statistical program (The R Foundation for Statistical Computing Platform, version 3.3.2.). Results are presented as mean and standard deviation (SD), median and interquartile range (IQR) or range as indicated. Categorical variables were compared using Fishers exact test. Numerical variables were compared with unpaired, two-tailed Student's T-test. Statistical significance is considered if $p$ was below 0.05 .

\section{Results}

Two hundred and thirty-one adult patients with AID were analyzed in the GARROD registry. After previous anti-inflammatory treatment options such as for instance NSAIDS, colchicine or glucocorticoids failed to control the systemic inflammation anti-IL-1 $\beta$ treatment was initiated and patients could be enrolled in the GARROD registry immediately or within the following 12 months. Demographic characteristics are 
indicated in Table 1. The mean age of patients with AID at symptom onset was 25.1 years. The time between the first AID symptoms and recruitment to the GARROD registry was 17.8 years. Mean age at baseline was 42.9 years.

Twenty-nine patients with AID (13\%) had primary or secondary AA amyloidosis at the time of initiation of the anti-IL1 $\beta$ treatment [Table 1].

\section{Autoinflammatory syndromes and anti-IL1 $\beta$ targeting treatment}

Patients with FMF $(n=71)$ comprised $30.7 \%$ of all individuals in the GARROD registry (Table 2$)$. They were also the largest patient group (53.4\%) with monogenetic AID (Table 3). Although anti-IL1 $\beta$ targeting medication has been approved and are standard of care for most patients with CAPS and TRAPS, both syndromes were less prevalent in this cohort. Patients with non-classified syndromes $(n=16)$ had inflammation of unknown origin but did not fulfil the diagnostic criteria for any known monogenetic or polygenetic AID. Patients with gout $(n=14)$, idiopathic (recurrent) pleuro-pericarditis $(n=8)$, Schnitzler's syndrome $(n=7)$ are indicated in Table 2. Patients with idiopathic AA amyloidosis $(n=6)$ had no primary inflammatory disease. Nephrotic syndrome due to AA amyloidosis was the first clinical symptom in this patient group. Miscellaneous AID and other inflammatory diseases $(n=15)$ were PFAPA $(n=3)$, Mevalonate Kinase Deficiency $(n=3)$, Cogan's syndrome $(n=2)$, osteomyelitis $(n=2)$, urticaria $(n=1)$, panniculitis $(n=1)$, Whipple's disease $(n=1)$, common variable immunodeficiency $(n=1)$, hemophagocytic syndrome $(n=1)$ and Erdheim-Chester disease $(n=1)$ (Table 2).

One-hundred and twenty-nine patients (56\%) had an onset of AID symptoms after the age of 18 years (Table 2). Patients with monogenetic AID (FMF, CAPS, TRAPS) tend to report a disease onset before the age of eighteen (63\%), whereas only $37 \%$ of polygenetic or unclassified AID symptoms started before the age of 18 years. Patients with unclassified AID were evenly distributed between juvenile and adult-onset. Polygenetic AID disease onset was more common in adults (Table 2).

\section{Clinical diagnosis and genetic testing for AID}

Molecular genetic analyses for monogenetic AIDs were performed in 128 of 133 patients (96\%) and compared with the clinical diagnosis of FMF $(n=71)$, CAPS $(n=43)$ and TRAPS $(n=19)$. Genetic variants were considered to be either pathogenic $(p)$, variants of unknown significance $(v)$, benign polymorphisms or wild type (w) (Table 3). The autosomal recessive type of FMF with two pathogenic MEFV variants, either homozygous or compound heterozygous, was genetically confirmed in $65 \%$ of patients with FMF. By including an autosomal dominant type of FMF with at least one FMF variant ( $p v, p w$, vv or vw), the rate of molecular genetic confirmation increased to $88 \%$ of patients with the clinical diagnosis of FMF (Table 3). Nine patients with a clinical FMF diagnosis (13\%) had no molecular genetic confirmation by MEFV analysis. 
CAPS and TRAPS are rare diseases with an autosomal dominant trait. However, pathogenic variants were confirmed only in $14 \%$ of NLRP3 and $47 \%$ of TNFRSF $1 A$ analyses. The detection of VUS (51\% in NLRP3, 47\% in TNFRSF1A, Table 3) might support the clinical diagnosis of a monogenetic AID which was sufficient to initiate anti-IL1 $\beta$ therapy.

Further analyses for the identification and characterization of pathogenic variants and VUS will be necessary for the interpretation of molecular genetic results in the future.

\section{MEFV variants correlate with FMF severity}

FMF is the most abundant AID in this cohort. The MEFV variants p.Met680lle/Val and p.Met694Val/Ile are well known pathogenic variants that are associated with a more severe FMF disease. Patients with two high penetrance variants are younger at FMF onset, have a higher FMF activity score and are more likely to develop secondary AA amyloidosis (Table 4). Patients with FMF + AA had a mean age of 17.3 years at FMF onset and 45.6 years at the diagnosis of $A A$ amyloidosis (Table 5 ). Patients with idiopathic AA amyloidosis seem to have no symptomatic primary inflammatory disease and develop AA amyloidosis at the same age of 44.2 years compared to patients with FMF + AA (Table 5).

\section{Discussion}

This is a multicenter retrospective analysis of 231 patients with AID who were treated with IL-1 $\beta$ blocking medication between 2013 and 2018. The mean age at baseline was 42.9 years which is consistent with the data from previously published registries $[11,16,17]$. In $56 \%$ of the patients the first inflammatory attacks occurred after the age of 18 years. The mean disease duration was 17.8 years between the symptom onset and the initiation of anti-IL-1 $\beta$ medication, highlighting an urgent need for diagnostic and therapeutic improvement.

The most prevalent diagnoses were FMF, CAPS and AOSD. When the GARROD registry was established in 2013, CAN and AKN were both approved only for CAPS. In the past years, AKN was widely used for rare AID in Germany without approval, probably because of the advantage of a short half-life of AKN, and to avoid the high costs of CAN. Only recently, CAN and AKN were both approved for SJIA and AOSD. A randomized controlled trial of CAN for patients with colchicine-resistant FMF, TRAPS and HIDS finally resulted in the approval of CAN for these rare diseases [18]. AKN has been investigated in an RCT in patients with crFMF [19]. Several investigators report the use of AKN in crFMF [20, 21]. Our data confirm the observations from previous reports and suggest that AKN can be used for patients with crFMF. Finally, AKN was approved for crFMF by the European Medical Agency (EMA) in April 2020.

Genetic analyses were performed in 129 patients with the clinical diagnoses of FMF, CAPS and TRAPS. Analysis of classified pathogenic variants led to genetic confirmation in FMF (65\%), CAPS (14\%) and TRAPS (47\%).

There is an ongoing controversy regarding the relevance of heterozygous variants in FMF, pathogenic variants and variants of unknown significance (VUS) in all monogenetic AID. 
If we consider VUS as low penetrance variants that might contribute to the inflammatory attacks, the rate of genetic confirmation increased by $+23 \%$ in FMF $(88 \%),+51 \%$ in CAPS $(65 \%)$ and $+47 \%$ in TRAPS (94\%). These data show a significant discrepancy between clinical diagnosis and molecular genetic confirmation of an AID, especially for rare diseases like CAPS and TRAPS.

The NLRP3 variants V198M and Q703K are considered as VUS or benign polymorphism $[13 ; 22 ; 23]$ and as low penetrance variants by others $[24 ; 25 ; 26]$. There is a controversy on the importance of the most prevalent TNFRSF1A variant R92Q. Some authors consider it as VUS [13] and others as low penetrance variant $[27 ; 28]$. However, the clinical phenotype should determine the diagnosis and if necessary, the treatment initiation of anti-IL1 $\beta$ drugs. If anti-IL $1 \beta$ treatment is effective to control clinical symptoms and systemic inflammation, the treatment regime should be continued even if the genetic test does not confirm the clinical diagnosis.

Our data confirm a dose dependent genotype phenotype correlation of high penetrance MEFV variants and a more severe FMF disease. These results are consistent with other reports in cohorts with pediatric [29] and adult patients [21, 30]. Our observations suggest the AID comprise a spectrum of disease which can present at any age. Therefore, pediatric and adult rheumatologists should be familiar with symptoms and differential diagnoses of various AIDs.

In our cohort FMF patients with AA amyloidosis had a mean disease duration of 28.3 years until the diagnosis of AA amyloidosis was established. Lack of information, limited compliance and incomplete control of the inflammatory attacks probably contribute to the risk of AA amyloidosis. However, even if we would be able to prevent AA amyloidosis in all AID patients, we would continue to observe patients with idiopathic AA amyloidosis [21].

Limitations of our study were the retrospective analysis which was partially dependent on the patients recall of their first attacks and the heterogeneous cohort with monogenetic and polygenetic AID with and without AA-amyloidosis.

Our data underline the importance for clinicians to learn the features of clinical presentation, differential diagnosis and treatment of AID. Our findings suggest that patients with AID should be guided by pediatric and adult rheumatologists, nephrologists and dermatologists. Networks of pediatric and adult AID specialists could help to increase the knowledge about the presentation of AID in small children, adolescents and adults.

\section{Abbreviations}

AA: Amyloid-A Amyloidosis

AID: Autoinflammatory Disease

AOSD: Adult-Onset Still's Disease 
CAPS: Cryopyrin-Associated Periodic Syndrome

crFMF: colchicine refractory FMF

FMF: Familial Mediterranean Fever

GARROD: German Anti-IL1 treatment RegistRy in Orphan Disease

TRAPS: Tumor Necrosis Factor Receptor-Associated Periodic Syndrome

VUS: Variant of Unknown Significance

\section{Declarations}

\section{Ethics approval and consent to participate}

This study reports on human data. Ethics approval was stated by the local ethics committee of the University of Heidelberg (reference S-103/2013).

Each individual provided written informed consent as approved by the ethics committee (reference S103/2013).

\section{Consent for publication}

Not applicable. The manuscript does not contain any individual persons data.

\section{Availability of data and materials}

Data sharing not applicable to this article as no datasets were generated or analyzed during the current study.

\section{Funding}

NB and contributing centers were funded by an unrestricted research grant by Swedish Orphan Biovitrum (SOBI). SOBI did not influence the design of the study. SOBI was not involved in collection, analysis, and interpretation of data. SOBI was not involved in the writing and revision of the manuscript.

\section{Competing interests}

NB received research grants from $\mathrm{SOBI}$ and Novartis, and speaking honoraria from $\mathrm{SOBI}$, Novartis, Roche, MSD, Abbott, Pfizer, Boehringer, Actelion and UCB. 
JR received research grants from $\mathrm{SOBI}$ and Novartis and speaking and consulting honoraria from Abbvie, Biogen, BMS, Chugai, GSK, Janssen, Lilly, MSD; Mylan, Novartis, Roche, Sanofi, Sobi, UCB.

MK received lecture honoraria from SOBI, Novartis, Roche, Sanofi and Medac

IK, $\mathrm{HT}, \mathrm{KK}, \mathrm{BK}, \mathrm{DK}, \mathrm{MN}, \mathrm{CH}, \mathrm{HML}$ declare that they have no competing interests.

\section{Authors' contributions}

NB formulated the concept, maintained and analyzed data. NB and MK drafted the manuscript. All authors contributed patient data to the GARROD registry, reviewed and approved the final manuscript.

\section{Acknowledgements}

Not applicable.

\section{References}

1. Livneh A, Langevitz P, Zemer D, Zaks N, Kees S, Lidar T, et al. Criteria for the diagnosis of familial Mediterranean fever. Arthritis Rheum 1997;40:1879-85.

2. Kuemmerle-Deschner JB, Ozen S, Tyrrell PN, Kone-Paut I, Goldbach-Mansky R, Lachmann H, et al. Diagnostic criteria for cryopyrin-associated periodic syndrome (CAPS). Ann Rheum Dis 2017;76:9427.

3. Federici S, Sormani MP, Ozen S, Lachmann HJ, Amaryan G, Woo P, et al.; Paediatric Rheumatology International Trials Organisation (PRINTO) and Eurofever Project. Evidence-based provisional clinical classification criteria for autoinflammatory periodic fevers. Ann Rheum Dis 2015;74:799-805.

4. Nirmala N, Brachat A, Feist E, Blank N, Specker C, Witt M, et al. Gene-expression analysis of adultonset Still's disease and systemic juvenile idiopathic arthritis is consistent with a continuum of a single disease entity. Pediatr Rheumatol Online J 2015;13:50.

5. Sighart R, Rech J, Hueber A, Blank N, Löhr S, Reis A, et al. Evidence for genetic overlap between adult onset Still's disease and hereditary periodic fever syndromes. Rheumatol Int 2018;38:111-20.

6. Yamaguchi M, Ohta A, Tsunematsu T, Kasukawa R, Mizushima Y, Kashiwagi H, et al. Preliminary criteria for classification of adult Still's disease. J Rheumatol 1992;19:424-30.

7. Fautrel B, Le Moël G, Saint-Marcoux B, Taupin P, Vignes S, Rozenberg S, et al. Diagnostic value of ferritin and glycosylated ferritin in adult onset Still's disease. J Rheumatol 2001;28:322-9.

8. Feist E, Mitrovic S, Fautrel B. Mechanisms, biomarkers and targets for adult-onset Still's disease. Nat Rev Rheumatol 2018;14:603-18.

9. Goldbach-Mansky R, Kastner DL. Autoinflammation: the prominent role of IL-1 in monogenic autoinflammatory diseases and implications for common illnesses. J Allergy Clin Immunol 
2009;124:1141-9.

10. Doherty TA, Brydges SD, Hoffman HM. Autoinflammation: translating mechanism to therapy. J Leukoc Biol 2011;90:37-47.

11. Colafrancesco S, Priori R, Valesini G, Argolini L, Baldissera E, Bartoloni E, et al. Response to Interleukin-1 Inhibitors in 140 Italian Patients with Adult-Onset Still's Disease: A Multicentre Retrospective Observational Study. Front Pharmacol 2017;8:369.

12. Kedor C, Listing J, Zernicke J, Weiß A, Behrens F, Blank N, et al. Canakinumab for Treatment of AdultOnset Still's Disease to Achieve Reduction of Arthritic Manifestation (CONSIDER): phase II, randomized, double-blind, placebo-controlled, multicenter, investigator-initiated trial. Ann Rheum Dis 2020;79:1090-7.

13. https://infevers.umai-montpellier.fr; last access 04-Jan-2021

14. Sipe JD, Benson MD, Buxbaum JN, Ikeda SI, Merlini G, Saraiva MJ, et al. Amyloid fibril proteins and amyloidosis: chemical identification and clinical classification International Society of Amyloidosis 2016 Nomenclature Guidelines. Amyloid 2016;23:209-13.

15. Blank N, Hegenbart U, Dietrich S, Brune M, Beimler J, Röcken C, et al. Obesity is a significant susceptibility factor for idiopathic AA amyloidosis. Amyloid 2018;25:37-45.

16. Giampietro C, Ridene M, Lequerre T, Costedoat Chalumeau N, Amoura Z, Sellam J, et al; CRI (Club Rhumatismes et Inflammation). Anakinra in adult-onset Still's disease: long-term treatment in patients resistant to conventional therapy. Arthritis Care Res 2013;65:822-6.

17. Proft F, Fleck M, Fiehn C, Schulze-Koops H, Witt M, Dörner T, et al. Efficacy and safety analysis of offlabel treatment with biologics in autoinflammatory diseases: Experiences from a German registry (GRAID2). Z Rheumatol 2018;77:46-54.

18. De Benedetti F, Gattorno M, Anton J, Ben-Chetrit E, Frenkel J, Hoffman HM, et al. Canakinumab for the Treatment of Autoinflammatory Recurrent Fever Syndromes. N Engl J Med 2018;378:1908-19.

19. Ben-Zvi I, Kukuy O, Giat E, Pras E, Feld O, Kivity S, et al. Anakinra for Colchicine-Resistant Familial Mediterranean Fever: A Randomized, Double-Blind, Placebo-Controlled Trial. Arthritis Rheumatol 2017;69:854-62.

20. Pecher AC, Igney-Oertel A, Kanz L, Henes J. Treatment of familial Mediterranean fever with anakinra in patients unresponsive to colchicine. Scand J Rheumatol 2017;46:407-9.

21. Köhler BM, Lorenz HM, Blank N. IL1-blocking therapy in colchicine-resistant familial Mediterranean fever. Eur J Rheumatol 2018;5:230-4.

22. Naselli A, Penco F, Cantarini L, Insalaco A, Alessio M, Tommasini A, et al. Clinical Characteristics of Patients Carrying the Q703K Variant of the NLRP3 Gene: A 10-year Multicentric National Study. J Rheumatol 2016;43:1093-100.

23. Lidar M, Brantz Y, Shinar Y, Reznik-Wolf H, Livneh A, Ben Zvi I, et al. A high and equal prevalence of the Q703K variant in NLRP3 patients with autoinflammatory symptoms and ethnically matched controls. Clin Exp Rheumatol 2017; 108:82-5. 
24. Kuemmerle-Deschner JB, Verma D, Endres T, Broderick L, de Jesus AA, Hofer F, et al. Clinical and Molecular Phenotypes of Low-Penetrance Variants of NLRP3: Diagnostic and Therapeutic Challenges. Arthritis Rheumatol 2017;69:2233-40.

25. Rowczenio DM, Gomes SM, Aróstegui JI, Mensa-Vilaro A, Omoyinmi E, Trojer $\mathrm{H}$, et al. Late-Onset Cryopyrin-Associated Periodic Syndromes Caused by Somatic NLRP3 Mosaicism-UK Single Center Experience. Front Immunol 2017;8:1410.

26. Vitale A, Lucherini OM, Galeazzi M, Frediani B, Cantarini L. Long-term clinical course of patients carrying the Q703K mutation in the NLRP3 gene: a case series. Clin Exp Rheumatol 2012;30:943-6.

27. Lachmann HJ, Papa R, Gerhold K, Obici L, Touitou I, Cantarini L, et al; Paediatric Rheumatology International Trials Organisation (PRINTO), the EUROTRAPS and the Eurofever Project. The phenotype of TNF receptor-associated autoinflammatory syndrome (TRAPS) at presentation: a series of 158 cases from the Eurofever/EUROTRAPS international registry. Ann Rheum Dis 2014;73:2160-7.

28. Ruiz-Ortiz E, Iglesias E, Soriano A, Buján-Rivas S, Español-Rego M, Castellanos-Moreira R, et al. Disease Phenotype and Outcome Depending on the Age at Disease Onset in Patients Carrying the R92Q Low-Penetrance Variant in TNFRSF1A Gene. Front Immunol 2017;8:299.

29. Kallinich T, Hinze $C$, Wittkowski H. Classification of autoinflammatory diseases based on pathophysiological mechanisms. Z Rheumatol 2020;79:624-38.

30. Tunca M, Akar S, Onen F, Ozdogan H, Kasapcopur O, Yalcinkaya F, et al.; Turkish FMF Study Group. Familial Mediterranean fever (FMF) in Turkey: results of a nationwide multicenter study. Medicine 2005;84:1-11.

\section{Tables}

Due to technical limitations, tables pptx is only available as a download in the Supplemental Files section.

\section{Supplementary Files}

This is a list of supplementary files associated with this preprint. Click to download.

- Tables.pptx 